P. S. Bullen, Department of Mathematics, University of British Columbia, Vanvouver BC, Canada V6T 1Z2,

e-mail address: bullen@unixg.math.ubc.ca

D. N. Sarkhel Department of Mathematics, University of Kalyani, Kalyani, W. B., India 741235

\title{
PROPERTIES OF DERIVATIVE-LIKE FUNCTIONS
}

\begin{abstract}
In this paper refinements and extensions of properties that give generalized derivatives the basic properties of ordinary derivatives are discussed; for instance the Darboux, Baire-1, Denjoy, Zahorski properties.
\end{abstract}

\section{Introduction}

It is known that a finite approximate derivative, (ap) $F^{\prime}=f: I=[a, b] \rightarrow \mathbb{R}, a<b$, shares many of the interesting properties of ordinary derivatives; ([5], [7], [9], [16]). Properties such as:

(i) the Baire-1 property,

(ii) the Darboux or intermediate value property,

(iii) the mean value property,

(iv) the Denjoy or Denjoy-Clarkson property: and in addition,

(v) $f(x)=F^{\prime}(x)$ on a dense open set in $I$.

Further: Weil [17] has strengthened (iv) to:

(vi) if $f^{-1}(] \alpha, \beta[) \neq \emptyset$, then $\left\{x ; x \in f^{-1}(] \alpha, \beta[), f(x)=F^{\prime}(x)\right\}$ has positive measure; and O'Malley [8] proved the surprisingly sharp property:

(vii) for every $x$ in $I$ there is an $x_{0}$ in $I$ such that $f(x)=F^{\prime}\left(x_{0}\right)$.

Weil [18] has also proved:

Key Words: Generalized derivatives, porosity, partitioning covers, Baire-1, interlocking, Zahorski, Darboux

Mathematical Reviews subject classification: Primary: 26A24

Received by the editors October 20, 1996 
(viii) $f$ has a certain property $Z$ on $I$, a property stronger than the Zahorski property $M_{3}[19]$.

It might be remarked that the Baire-1 property often seems crucial in the deduction of the other properties.

In a welcome attempt to reveal how the various properties arise Bruckner, O'Malley \& Thomson [2] have studied the unified notion of path derivatives, and Thomson [15] has studied the more general notion of derivatives relative to simple systems, proving various properties of derivatives under the external intersection condition, (E.I.C. $[\mathrm{m}]$ ), the intersection condition, (I.C.), and the non-porosity condition. The Baire-1 property in particular is obtained in $[2,6.3$, p. 113] under (E.I.C. [m]), and in [15] under a wider condition [15, Lemma 9.7, p. 358]; the Darboux property is proved by assuming the Baire-1 property [2, 6.4, p. 113], [15, 8.1, p. 352].

In this paper we show, amongst other things, that a semi-Baire-1 property and all the properties (ii) through (viii) in stronger and more revealing forms extend to functions like derivatives relative to limiting systems, (essentially two-sided simple systems [15, p. 280]), under an interlocking condition wider than (I.C.), and often a generalized non-porosity condition. The Baire-1 property may however fail even under stronger conditions. This and other critical aspects of the present theory are illustrated by appropriate examples.

\section{Definitions and Lemmas}

Throughout we suppose that $F, f: I=[a, b] \rightarrow \mathbb{R}, a<b$ and $T$ is a fixed limiting system on $\mathbb{R}$, by which we mean an arbitrary collection $T=\{T(x) ; x \in \mathbb{R}\}$ where each $T(x)$ is a non-empty family of subsets of $\mathbb{R}$ such that, if $A \in T(x)$, then $x \in A$ and $x$ is a two-sided limit point of $A$, and $A \cap] c, d[\in T(x)$ for all $c, d, c<x<d$.

For each $\tau(x) \in T(x), x \in I$, we denote by $I_{\tau(x)}$ the family of all closed intervals $[u, v], u \leq v$ with $u$ in $\tau(x) \cap[a, x]$ and $v$ in $\tau(x) \cap[x, b]$. We define the extreme $T$-derivates of $F$ on $I$ by

$$
\begin{aligned}
& T \bar{F}^{\prime}(x)=\inf _{\tau(x) \in T(x)}\left[\sup \left\{\frac{F(v)-F(u)}{v-u} ;[u, v] \in I_{\tau(x)}, u<v\right\}\right], \\
& T \underline{F}^{\prime}(x)=\sup _{\tau(x) \in T(x)}\left[\inf \left\{\frac{F(v)-F(u)}{v-u} ;[u, v] \in I_{\tau(x)}, u<v\right\}\right] .
\end{aligned}
$$

Clearly $T(\overline{-F})^{\prime}(x)=-T \underline{F}^{\prime}(x)$. If $T \bar{F}^{\prime}(x) \leq T \underline{F}^{\prime}(x)$, then $F$ is said to be $T$-derivable at the point $x$. If $T \bar{F}^{\prime}(x)=T \underline{F}^{\prime}(x)$, then this common value is called the $T$-derivative of $F$ at $x, T F^{\prime}(x)$.

As well as finite or infinite $T$-derivatives we will also study $T$-derivative-like functions; that is, functions $g$ that satisfy $T \bar{F}^{\prime}(x) \leq g(x) \leq T \underline{F}^{\prime}(x)$. 
Remark 2.1 In the absence of other conditions we can have that $T \bar{F}^{\prime}(x)<T F^{\prime}(x)$. This cannot happen if $T$ is filtering down; that is, if the intersection of two members of $T(x)$ is also a member of $T(x)$. However we do not assume this condition.

Remark 2.2 The definition of the T-derivative is unique, but this is not true for the simple-derivatives. The simple system naturally associated with $T$ is $S=\{S(x) ; x \in$ $\mathbb{R}\}$, where $S(x)=\{E ; \mathbb{R} \supseteq E \supseteq \tau(x) \in T(x)\}\}$. Then, according to Thomson $[15$, p. 281], $F$ has an $S$-derivative $\alpha$ at a point $x$ in $I$ if for every $\eta>0$ there is a $\tau(x) \in T(x)$ such that

$$
|F(y)-F(x)-\alpha(y-x)| \leq \eta|y-x|
$$

for all $y$ in $\tau(x) \cap I$. Clearly then $F$ is $T$-derivable at $x$ and $T \bar{F}^{\prime}(x) \leq \alpha \leq T \underline{F}^{\prime}(x)$, but the converse is not true.

Remark 2.3 The weakest limiting system is $T_{1}$, where each $T_{1}(x)$ consists of all sets containing $x$ and having $x$ as a two-sided limit point. Clearly $T_{1} \bar{F}^{\prime}(x)=\max \left\{D_{+} F(x), D_{-} F(x)\right\}$, and $T_{1} \underline{F}^{\prime}(x)=\min \left\{D^{+} F(x), D^{-} F(x)\right\}$, with obvious modifications if $x=a, b$. Darboux and mean value properties of $T_{1}$-derivative-like functions have been studied in Sarkhel \& Seth [12], and Bullen \& Sarkhel [4].

Our main line of reasoning will be Baire's theorem [10, (9.2), p. 54] and the partitioning property, which has many other interesting applications. (See [3] and [14].)

Let $E \subseteq X \subseteq I$. Following Sarkhel \& $\operatorname{Kar}[11, \S 2]$, a sequence of sets $\left\{E_{n}\right\}$ whose union is $E$ is called an $E$-form with parts $E_{n}$; if further each part $E_{n}$ is closed in $E$, then the $E$-form is said to be closed. An increasing $E$-form is called an $E$-chain. By a perfect portion of $E$ we shall mean a section $E \cap[p, q]$ that is perfect and contains both $p$ and $q$.

Every choice $\tau=\{\tau(x) \in T(x) ; x \in X\}$ is called a $T$-full cover of $E$. A finite, possibly empty, family $\varpi=\left\{\left(\left[u_{j}, v_{j}\right], x_{j}\right) ;\left[u_{j}, v_{j}\right] \in I_{\tau\left(x_{j}\right)}\right\}$ is called a $\tau$-partition over $E($ in $I)$ if for all $j$ the intervals $] u_{j}, v_{j}$ [ are pairwise disjoint, and $x_{j} \in E$; if further $\bigcup_{j}\left[u_{j}, v_{j}\right]=E$, then $\varpi$ is called a $\tau$-partition of $E$. We put

$$
\begin{aligned}
|\varpi| & =\sum_{j}\left(v_{j}-u_{j}\right), \quad \sum(f, \varpi)=\sum_{j} f\left(x_{j}\right)\left(v_{j}-u_{j}\right), \\
\Delta(F, \varpi) & =\sum_{j}\left(F\left(v_{j}\right)-F\left(u_{j}\right)\right),
\end{aligned}
$$

where all these sums are to be zero if $\varpi=\emptyset$.

Given a neighborhood $] x_{1}, x_{2}$ [ of each point $x \in E$ we will call $\{\tau(x) \cap$ ]$x_{1}, x_{2}[\}$ a refinement of $\tau$ on $E$. 
Lemma 2.1 Let $\tau$ be a $T$-full cover of a set $E \subseteq I$. If $E$ is of measure zero, then for every $\epsilon>0$ there is a refinement $\lambda$ of $\tau$ on $E$ such that for every $\lambda$-partition $\varpi$ over $E$ we have $\sum(|f|, \varpi)<\epsilon$ and $|\varpi|<\epsilon$.

Proof. Since $|E|=0$, there are open sets $G_{n} \supseteq E$ with $\left|G_{n}\right|<n^{-1} 2^{-n} \epsilon, n=$ $1,2,3, \ldots$. It then suffices to take a refinement $\lambda$ on $E$ such that $\lambda(x)$ is contained in a component of $G_{n}$ if $n-1 \leq|f(x)|<n$.

We say that $T$ is partitioning, or has the partitioning property on $I$ if every $T$-full cover $\tau$ of $I$ induces a $\tau$-partition of $I$.

Lemma 2.2 If $T$ is partitioning on $I$, then it is also partitioning on every subinterval $[c, d], c<d$, of $I$.

Proof. Let $\lambda$ be any $T$-full cover of $[c, d]$. For each $x \in I \backslash[c, d]$ select $\tau(x) \in T(x)$ such that $\tau(x) \subseteq]-\infty, c[$ or $\tau(x) \subseteq] d, \infty[$; also let $\tau(c)=\lambda(c), \tau(d)=\lambda(d)$, and $\tau(x)=\lambda(x) \cap] c, d[$ for $c<x<d$. Then $\tau$ is a $T$-full cover of $I$, and hence there is a $\tau$-partition of $I$ which clearly induces a $\lambda$-partition of $[c, d]$.

The partitioning property arises from various intersection conditions, [2]. Following [2] and [15], we say that $T$ satisfies the intersection condition, (I.C.), on $I$ if for every $T$-full cover $\tau$ of $I$ there are $\delta_{x}>0, x \in I$, such that if $x, y \in I$ and $0<y-x<\min \left\{\delta_{x}, \delta_{y}\right\}$, then $\tau(x) \cap \tau(y) \cap[x, y] \neq \emptyset$. If $A_{n}=\left\{x \in I ; \delta_{x}>n^{-1}\right\}$, then $\left\{A_{n}\right\}$ is an I-chain. If $x, y \in A_{n}$ and $0<y-x<n^{-1}$, then $\tau(x)$ and $\tau(y)$ intersect as above and so $[x, y]$ has a $\tau$-partition of the form $\{([x, u], x),([u, y], y)\}$. We generalize this to the interlocking property, ILP, in terms of the interlocking condition, $I L C$, as follows.

We say $\tau$ satisfies $I L C$ on a set $A \subseteq I$ if for any two points $x<y$ in $A$ there is a set $E \subset] x, y$ [, of measure zero, such that for any refinement $\lambda$ of $\tau$ on $E$ there is a $\tau$-partition of $[x, y]$ of the form $\{([x, u], x),([v, y], y)\} \cup \varpi$, where $\varpi$ is a $\lambda$-partition of $[u, v]$ over $E$.

Then $T$ is said to satisfy ILP on $I$ if for every $T$-full cover $\tau$ of $I$ there is an $I$-chain $\left\{A_{n}\right\}$ such that for each $n$ there is a $\delta_{n}>0$ such that $\tau$ satisfies ILC on $A_{n} \cap J$ for every closed interval $J$ with $|J|<\delta_{n}$. We then say that $\tau$ satisfies ILP on I via $\left\{\left(A_{n}, \delta_{n}\right)\right\}$.

The following result extends (4.7.3) of [2, p. 109].

Lemma 2.3 The interlocking property implies the partitioning property.

Proof. Let an arbitrary $T$-full cover $\tau$ of $I$ satisfy ILP on $I$ via $\left\{\left(A_{n}, \delta_{n}\right)\right\}$. Let $E$ denote the set of points $x$ of $I$ such that every neighborhood of $x$ in $I$ contains a closed interval admitting no $\tau$-partition. Then $E$ is clearly closed, and it is easily seen that the closure of every interval contained in $I \backslash E$ has a $\tau$-partition. Hence $E$ is perfect, and we need only show that $E$ is empty. 
Suppose that $E \neq \emptyset$. Then by Baire's theorem some $A_{m}$ must be dense in some perfect portion, $E \cap[c, d]$ of $E$, with $d-c<\delta_{m}$.

Let $c \leq u<v \leq d$. If $E \cap] u, v[=\emptyset$, then as remarked above $[u, v]$ has a $\tau$ partition. If $E \cap] u, v\left[\neq \emptyset\right.$ put $u_{1}=\inf (E \cap] u, v[)$ and $v_{1}=\sup (E \cap] u, v[)$. Then $u_{1}, v_{1} \in E$ and $u \leq u_{1}<v_{1} \leq v$. Since $\left.E \cap\right] u, u_{1}[=\emptyset=E \cap] v_{1}, v[$, both $\left[u, u_{1}\right],\left[v_{1}, v\right]$ have $\tau$-partitions, and because $E$ is perfect $u_{1}, v_{1}$ must be limit points of $E \cap] u_{1}, v_{1}\left[\right.$, and hence of $\left.A_{m} \cap\right] u_{1}, v_{1}\left[\right.$. Since $\left\{A_{n}\right\}$ is an $I$-chain, there is an $A_{n} \supseteq A_{m}$ such that $u_{1}, v_{1} \in A_{n}$. Then there are points $x, y \in A_{m}, u_{1}<x<y<v_{1}$, such that $x-u_{1}<\delta_{n}$ and $v_{1}-y<\delta_{n}$. Since $u_{1}, x, y, v_{1} \in A_{n}$, there are, by ILC, $\tau$ partitions of both $\left[u_{1}, x\right]$, and $\left[y, v_{1}\right]$. Also since $x, y \in A_{m}$ and $0<y-x<d-c<\delta_{m}$ we have, by ILC, a $\tau$-partition of $[x, y]$. Hence it follows that every $[u, v] \subset] c, d[$ has a $\tau$-partition, and this contradicts the condition $E \cap] c, d[\neq \emptyset$.

As observed by Thomson [15, p. 420], set porosity is precisely the right notion to capture certain properties of generalized derivatives. The porosity of a set $A$ at a point $x$ is the number

$$
p(A, x)=\limsup _{\delta \rightarrow 0+}\left[\sup \left\{\frac{(v-u)}{\delta} ;\right] u, v[\subseteq] x-\delta, x+\delta[\backslash(A \cup\{x\})\}\right] .
$$

We set $p_{T}(x)=\sup \{p(A, x) ; A \in T(x)\}$, and if $p_{T}(x)=0$ for all $x \in I$, then $T$ is said to be non-porous on $I$.

We say that $T$ is non-porous in the generalized sense on $I,(\mathrm{NPG})$, if there is a closed $I$-form $\left\{E_{n}\right\}$ such that for each $n$ we have that $\sup \left\{p_{T}(x) ; x \in E_{n}\right\}<1$.

Note The properties ILP and (NPG) are hereditary.

The following lemma greatly extends Theorem 4.4 of [2, p. 106].

Lemma 2.4 Hypotheses: $x \in E \subseteq I, E$ is closed, $p_{T}(x)<1, F$ is monotone on the closure of each component of $I \backslash E, \alpha \in \mathbb{R}$ and $M=\max \left\{\alpha, T \bar{F}^{\prime}(x)\right\} ; m=$ $\min \left\{\alpha, T \underline{F}^{\prime}(x)\right\}$.

Conclusions: (i) If $F(v)-F(u) \leq \alpha(v-u)$ for all $u, v \in E, u \leq x \leq v$, then

$$
\bar{F}^{\prime}(x) \leq\left\{\begin{array}{l}
M /\left(1-p_{T}(x)\right) \quad \text { if } M \geq 0, \\
M\left(1-p_{T}(x)\right) \quad \text { if } M \leq 0 ;
\end{array}\right.
$$

if further $x$ is a limit point of $E$, then

$$
T \underline{F}^{\prime}(x) \leq \begin{cases}\alpha /\left(1-p_{T}(x)\right) & \text { if } T \underline{F}^{\prime}(x) \geq 0 \\ \alpha\left(1-p_{T}(x)\right) & \text { if } T \underline{F}^{\prime}(x) \leq 0 .\end{cases}
$$

(ii) If $F(v)-F(u) \geq \alpha(v-u)$ for all $u, v \in E, u \leq x \leq v$, then

$$
\underline{F}^{\prime}(x) \geq \begin{cases}m /\left(1-p_{T}(x)\right) & \text { if } m \leq 0 \\ m\left(1-p_{T}(x)\right) & \text { if } m \geq 0\end{cases}
$$


if further $x$ is a limit point of $E$, then

$$
T \bar{F}^{\prime}(x) \geq \begin{cases}\alpha /\left(1-p_{T}(x)\right) & \text { if } T \bar{F}^{\prime}(x) \leq 0, \\ \alpha\left(1-p_{T}(x)\right) & \text { if } T \bar{F}^{\prime}(x) \geq .\end{cases}
$$

Note If $E=\{x\}$, then we can take any $\alpha$ in (i) and (ii). Hence, clearly, if $F$ is non-decreasing on $I$, then

$$
T \underline{F}^{\prime}(x)\left(1-p_{T}(x)\right) \leq \underline{F^{\prime}}(x) \text { and } \bar{F}^{\prime}(x) \leq \frac{T \bar{F}^{\prime}(x)}{1-p_{T}(x)},
$$

with equality holding if $p_{T}(x)=0$.

Proof. (i) First, ignoring the trivial case $M=\infty$, let $p_{T}(x)<q<1$ and $M<\beta$, where $\beta<0$ if $M<0$. Suppose $a \leq x<b$. Then there are $\tau(x) \in T(x)$ and $t \in \tau(x) \cap] x, b[$ such that

$$
\begin{aligned}
F(y)-F(x) \leq \beta(y-x) & \text { if } \quad y \in(E \cup \tau(x)) \cap[x, t], \\
d-c<q(d-x) & \text { if } \quad] c, d[\subseteq] x, t[\backslash \tau(x), c>x .
\end{aligned}
$$

Consider now any $y \in] x, t[\backslash(E \cup \tau(x))$, if there are any. Let $] r, s[$ be the component of $] x, t[\backslash E$ that contains $y$. Put

$$
c=\sup ((E \cup \tau(x)) \cap[r, y[) \text { and } d=\inf ((E \cup \tau(x)) \cap] y, s]) .
$$

Then $x \leq r \leq c \leq y \leq d \leq s \leq t, c>x$. Since, by (2), $d-y<q(d-x)$, there is a $v \in(E \cup \tau(x)) \cap[d, s]$ such that $v-y<q(v-x)$; and so $(1-q)(v-x)<y-x$. Again by (2), $y-c<q(y-x)$ and hence there is a $u \in(E \cup \tau(x)) \cap[r, c]$ such that $y-u<q(y-x)$, whence $(1-q)(y-x)<u-x$.

Now by hypothesis, $F$ is either non-decreasing or non-increasing on $[r, s]$. In the first case, by the above and (1) $F(y)-F(x) \leq F(v)-F(x) \leq \beta(v-x)$, whence

$$
F(y)-F(x) \begin{cases}<\beta(y-x) /(1-q) & \text { if } \beta>0 \\ \leq \beta(y-x) & \text { if } \beta<0 .\end{cases}
$$

In the second case, by the above and (1), $F(y)-F(x) \leq F(u)-F(x) \leq \beta(u-x)$, whence

$$
F(y)-F(x) \begin{cases}\leq \beta(y-x) & \text { if } \beta>0, \\ <\beta(1-q)(y-x) & \text { if } \beta<0 .\end{cases}
$$


These, in conjunction with (1), clearly prove

$$
D^{+} F(x) \leq \begin{cases}\beta /(1-q) & \text { if } \beta>0 \\ \beta(1-q) & \text { if } \beta<0\end{cases}
$$

By symmetry, if $a<x \leq b$, then

$$
D^{-} F(x) \leq \begin{cases}\beta /(1-q) & \text { if } \beta>0 \\ \beta(1-q) & \text { if } \beta<0\end{cases}
$$

So

$$
\bar{F}^{\prime}(x) \leq \begin{cases}\beta /(1-q) & \text { if } \beta>0, \\ \beta(1-q) & \text { if } \beta<0 .\end{cases}
$$

Letting $q \rightarrow p_{T}(x)+$ and $\beta \rightarrow M+$, the first two results follow. Next, let $x$ be a limit point of $E$ on the right. Ignoring the trivial case when $T \underline{F}^{\prime}(x)=-\infty$, take $\gamma<T \underline{F}^{\prime}(x)$, where $\gamma>0$ if $T \underline{F}^{\prime}(x)>0$. Then there is $\lambda(x) \in T(x)$ and $\left.e \in E \cap\right] x, b[$ such that

$$
\begin{aligned}
F(y)-F(x) & \geq \gamma(y-x) \text { if } y \in \lambda(x) \cap[x, e], \\
s-r<q(s-x) & \text { if }] r, s[\subseteq] x, e[\backslash \lambda(x), r>x .
\end{aligned}
$$

Now, fix $t \in \lambda(x) \cap] x, e[$. If $t \in E$, then by hypothesis and (3) $\alpha(t-x) \geq F(t)-F(x) \geq$ $\gamma(t-x)$, from which $\alpha \geq \gamma$.

If $t \notin E$, let $] r, s$ [ be the component of ] $x, e[\backslash E$ containing $t$. Then $r, s \in E$ and $x<r<t<s \leq e$. Let $c=\inf (\lambda(x) \cap] r, t])$ and $d=\sup (\lambda(x) \cap[t, s[)$. Since by (4), $c-r<q(c-x)$, there is a $u \in \lambda(x) \cap[c, t]$ such that $u-r<q(u-x)$, whence $(1-q)(u-x)<r-x$. Again by (4) $s-d<q(s-x)$, and hence there is a $v \in \lambda(x) \cap[t, d]$ such that $s-v<q(s-x)$, whence $(1-q)(s-x)<v-x$.

Now by hypothesis, $F$ is either non-increasing or non-decreasing on $[r, s]$. In the first case, by hypothesis and by the above and (3)

$$
\alpha(r-x) \geq F(r)-F(x) \geq F(u)-F(x) \geq \gamma(u-x)
$$

whence $\alpha(r-x)>\gamma(r-x) /(1-q)$ or $\alpha>\gamma /(1-q)$ if $\gamma<0$, and $\alpha(r-x) \geq \gamma(r-x)$ or $\alpha \geq \gamma$ if $\gamma>0$. In the second case, by hypothesis and by the above and (3)

$$
\alpha(s-x) \geq F(s)-F(x) \geq F(v)-F(x) \geq \gamma(v-x),
$$

whence $\alpha(s-x) \geq \gamma(s-x)$ or $\alpha \geq \gamma$ if $\gamma<0$, and $\alpha(s-x)>\gamma(s-x)(1-q)$ or $\alpha>\gamma(1-q)$ if $\gamma>0$. 
Thus we always have $\alpha \geq \gamma /(1-q)$ if $\gamma<0$ and $\alpha \geq \gamma(1-q)$ if $\gamma>0$. Letting $\gamma \rightarrow T \underline{F}^{\prime}(x)$ and $q \rightarrow p_{T}(x)+$, the second two results follow.

A similar proof holds if $x$ is a limit point of $E$ on the left.

(ii) This follows form (i) applied to $-F$.

Generalizing a well-known property of Baire-1 functions, we shall say that the function $f$ is semi-Baire- 1 on $I$ if for every $\alpha \in f(I)$ the level set $f^{-1}(\alpha)$ contains a point of continuity of $f$ relative to the closure of $f^{-1}(\alpha)$.

Finally we recall that a point $x \in I$ is termed a point of absolute continuity of the function $F$, an AC-point, if $x$ has a neighborhood in $I$ on which $F$ is AC. Also $F$ is called (ACG) on $I$ if $F$ is AC on each part of some closed $I$-form.

The following examples illustrate some of our ideas.

Example 2.5 Every bilateral system of paths $\left[2\right.$, p. 100] $P=\left\{P_{x} ; x \in \mathbb{R}\right\}, x \in$ $P_{x} \subseteq \mathbb{R}$ and $x$ a two-sided limit point of $P_{x}$, generates a limiting system $T_{P}$ which is defined by $T_{P}(x)=\left\{P_{x} \cap\right] c, d[; c<x<d\}$, which is filtering down. The notions of $P$-derivative and $T_{P}$-derivative coincide. If $P$ satisfies (I.C.), then $T_{P}$ satisfies (I.C.), and hence also ILP. If $P$ is non-porous, then so is $T_{P}$.

Example 2.6 If $T_{a p}(x)$ is the family of all measurable sets containing $x$, and having density 1 at $x$, then $T_{a p}$ is a filtering down, non-porous limiting system satisfying (I.C.), [2, p. 102]. The notions of approximate derivative and $T_{a p}$-derivative coincide.

Example 2.7 For each $\alpha>0$, we construct a limiting system $T_{\alpha}$ which is nonporous, and satisfies ILP but not (I.C.), and is or is not filtering down according as $\alpha$ is rational or not.

Define

$$
T_{\alpha}(x)= \begin{cases}\{] c, d[; c<x<d\} & \text { if } x \in \mathbb{Q}, \text { and } \\ \{] c, d[\cap(x+t \mathbb{Q}) ; c<x<d, t=1, \alpha\} & \text { if } x \text { is irrational. }\end{cases}
$$

Clearly, $T_{\alpha}$ is a non-porous limiting system.

Let now $\tau$ be any $T_{\alpha}$-full cover of $I$. Let $A_{n}=\{x \in I ; \tau(x)$ is dense in $] x-n^{-1}, x+$ $n^{-1}[\}, n=1,2, \ldots$ Clearly $\left\{A_{n}\right\}$ is an I-chain. Let $x, y \in A_{n}$ and $0<y-x<n^{-1}$. Fix a rational $r \in] x, y[$. If $\lambda$ is any refinement of $\tau$ on $\{r\}$, then $\lambda(r)$ is a neighborhood of $r$. Also, $\tau(x)$ is dense in $] x, r[$ and $\tau(y)$ is dense in $] r, y[$. So $\lambda(r)$ intersects both $\tau(x) \cap] x, r[$ and $\tau(y) \cap] r, y\left[\right.$. Hence, plainly, $\tau$ satisfies ILP on $I$ via $\left\{\left(A_{n}, n^{-1}\right)\right\}$. Thus $T_{\alpha}$ satisfies ILP.

But, consider any $I$-chain $\left\{E_{n}\right\}$. Obviously some $E_{n}$ must contain an uncountable set $B$ of irrationals. Then some $\xi \in B$ must be such that every neighborhood of $\xi$ contains uncountably many points of $B ;[6$, p. 129]. Since $\xi+\mathbb{Q}+\alpha \mathbb{Q}$ is countable, every neighborhood of $\xi$ contains points $\eta \in B$ such that $\eta \notin \xi+\mathbb{Q}+\alpha \mathbb{Q}$. This means 
that $[(\xi+\mathbb{Q}) \cup(\xi+\alpha \mathbb{Q})] \cap[(\eta+\mathbb{Q}) \cup(\eta+\alpha \mathbb{Q})]=\emptyset$. Since $\xi, \eta \in B \subseteq E_{n}$, it follows that $T_{\alpha}$ cannot satisfy (I.C.) on I.

Lastly, if $\alpha$ is rational, then $x+t \mathbb{Q}=x+\mathbb{Q}$ for $t=1, \alpha$; but if $\alpha$ is irrational, then $(x+\mathbb{Q}) \cap(x+\alpha \mathbb{Q})=\{x\}$. Hence clearly $T_{\alpha}$ is filtering down if $\alpha$ is rational, but not if $\alpha$ is irrational.

\section{Main Results}

We begin with a monotonicity theorem.

Theorem 3.1 Suppose that $T$ is partitioning on $I$.

(i) If $T \bar{F}^{\prime}(x)<\infty$ on I and $T \bar{F}^{\prime} \leq \alpha$ a.e. on $I$, then $F(x)-\alpha x$ is non-increasing on $I$.

(ii) If $T \underline{F}^{\prime}(x)>-\infty$ on $I$ and $T \underline{F}^{\prime} \geq \beta$ a.e. on $I$, then $F(x)-\beta x$ is non-decreasing on $I$.

Proof. (i) Let $A=\left\{x \in I ; T \bar{F}^{\prime}(x) \leq \alpha\right\}, E=I \backslash A$, and $\epsilon>0$. Assuming that $f(x)>T \bar{F}^{\prime}(x)$ for all $x \in I$, with $f(x)=\alpha+\epsilon$ for $x \in A$, there is a $T$-full cover $\tau$ of $I$ such that

$$
F(v)-F(u) \leq f(x)(v-u) \text { for all }[u, v] \in I_{\tau(x)}, x \in I .
$$

Again, since $|E|=0$, by (2.1), $\tau$ has a refinement $\lambda$ on $I$ such that for every $\lambda$-partition $\varpi_{0}$ over $E$ we have $\sum\left(|f|, \varpi_{0}\right)<\epsilon$ and $\left|\varpi_{0}\right|<\epsilon$.

Now let $a \leq c<d \leq b$. By (2.2) $[c, d]$ has a $\lambda$-partition, say $\varpi$. Then $\varpi=\varpi_{1} \cup \varpi_{0}$ where $\varpi_{1}$ and $\varpi_{0}$ are $\lambda$-partitions, (and so $\tau$-partitions), over $A$ and $E$ respectively. Hence, recalling the choice of $f, \tau$ and $\lambda$, we have

$$
\begin{aligned}
F(d)-F(c)=\Delta(F, \varpi) & \leq \sum(f, \varpi)=\sum\left(f, \varpi_{1}\right)+\sum\left(f, \varpi_{0}\right) \\
& \leq(\alpha+\epsilon)\left|\varpi_{1}\right|+\sum\left(|f|, \varpi_{0}\right) \\
& <\alpha(d-c)-\alpha\left|\varpi_{0}\right|+\epsilon\left|\varpi_{1}\right|+\epsilon \\
& \leq \alpha(d-c)+|\alpha| \epsilon+\epsilon|I|+\epsilon .
\end{aligned}
$$

Letting $\epsilon \rightarrow 0+$ we get $F(d)-F(c) \leq \alpha(d-c)$, which proves (i).

(ii) This follows by applying (i) to $-F$.

Next we prove the fundamental theorem of this paper.

Theorem 3.2 Suppose $T$ satisfies ILP on $I$, and $T \bar{F}^{\prime}(x)<\infty$ and $T \underline{F}^{\prime}(x)>-\infty$ for all $x \in I$. Then: 
(i) F lies between its one-sided extreme limits on either side, everywhere in I,

(ii) $F$ is (ACG) on $I$, it is Darboux on $I$, (ap) $F^{\prime}(x)$ exists finitely a.e. on $I$, and $F^{\prime}(x)$ exists finitely a.e. on a dense open set in $I$,

(iii) if $F$ is of bounded variation, $V B$, on a closed set $X \subseteq I$ and $F \mid X$ is continuous, then $F$ is $A C$ on $X$,

(iv) either $F$ is strictly monotonic and $A C$ on $I$, or $F$ has a local extremum at some $A C$-point of $F$ on $] a, b[$.

Note Other variants of (iv) appear in [12, p. 14] and [4, p. 227].

Proof. Part (i) is obvious. For (ii), assuming $f(x)>T \bar{F}^{\prime}(x)$ and $-f(x)<T \underline{F}^{\prime}(x)$, there are $T$-full covers $\tau, \lambda$ of $I$ such that

$$
\begin{aligned}
& F(v)-F(u) \leq f(x)(v-u) \text { for all }[u, v] \in I_{\tau(x)}, x \in I, \\
& F(v)-F(u) \geq-f(x)(v-u) \text { for all }[u, v] \in I_{\lambda(x)}, x \in I .
\end{aligned}
$$

Let $\tau, \lambda$ satisfy ILP on $I$ via $\left\{\left(A_{n}, \delta_{n}\right)\right\},\left\{\left(B_{n}, \eta_{n}\right)\right\}$ respectively. Put

$$
E_{n}=\left\{x \in A_{n} \cap B_{n} ;|f(x)| \leq n\right\}, \quad \rho_{n}=\min \left\{\delta_{n}, \eta_{n}\right\} .
$$

Then $\left\{E_{n}\right\}$ is an $I$-chain and both $\tau, \lambda$ satisfy ILP on $I$ via $\left\{\left(E_{n}, \rho_{n}\right)\right\}$.

Let $x, y \in E_{n}, 0<y-x<\rho_{n}$, and $\left.Z \subset\right] x, y[$ be the zero measure set as required by ILC of $\tau$. By (2.1), given $\epsilon>0$ there is a refinement $\mu$ of $\tau$ on $Z$ such that for every $\mu$-partition $\varpi$ over $Z$ we have $\sum(|f|, \varpi)<\epsilon$. By ILC of $\tau$, there is a $\tau$-partition $\{([x, u], x),([v, y], y)\} \cup \varpi$ of $[x, y]$ where $\varpi$ is a $\mu$-partition of $[u, v]$. Then by the choice of $f$ and $\tau$ we have

$$
\begin{aligned}
F(y)-F(x) & =(F(u)-F(x))+\Delta(F, \varpi)+(F(y)-F(v)) \\
& \leq f(x)(u-x)+\sum(f, \varpi)+f(y)(y-v) \\
& <n(u-x)+\epsilon+n(y-v) .
\end{aligned}
$$

Hence $F(y)-F(x) \leq n(y-x)$. Similarly, from ILC of $\lambda, F(y)-F(x) \geq-n(y-x)$. Hence $|F(y)-F(x)| \leq n(y-x)$, and $F \mid E_{n}$ is continuous.

Now, for any $n$, let $J_{n}$ be any closed interval with $0<\left|J_{n}\right|<\rho_{n}$, and let $x_{1}<y_{1}$ be any two points in the closure of $E_{n} \cap J_{n}$. There is $E_{m} \supseteq E_{n}$ such that $x_{1}, y_{1} \in E_{m}$. Since $0<y_{1}-x_{1}<\rho_{n}$ and $F \mid E_{m}$ is continuous, choosing points $x, y \in E_{n}$ close to $x_{1}, y_{1}$ respectively it follows at once from above that $\left|F\left(y_{1}\right)-F\left(x_{1}\right)\right| \leq n\left(y_{1}-x_{1}\right)$.

These Lipschitz conditions evidently imply that $F$ is (ACG) on $I$. Hence (ap) $F^{\prime}(x)$ exists finitely a.e. on $I,\left[10\right.$, p. 223 infra], and $F^{\prime}(x)$ exists finitely a.e. on a dense open set in $I$ (since by Baire's theorem the AC-points of $F$ are dense in $I$ ). Again, 
since $F$ is (ACG) on $I$ it is Baire- 1 on $\mathrm{I}$, which by (i) implies that $F$ is Darboux on I, [13, Theorem III, p. 21], [1, Theorem 6.1, p. 103].

(iii) This follows from $[10,(6.7)$, p. 227], since being (ACG) $F$ satisfies Lusin's condition $(\mathrm{N})$ on $I,[10,(6.1)$, p. 225].

(iv) Let $E$ denote the set of points of $I$ having no neighborhood in $I$ on which $F$ is strictly monotonic. Clearly $E$ is closed. Routine arguments shows that $F$ is strictly monotone on every component $] u, v[$ of $] a, b[\backslash E$, and then (i) implies that $F$ is strictly monotone and continuous on $[\mathrm{u}, \mathrm{v}]$, and hence by (iii) $F$ is $\mathrm{AC}$ on $[u, v]$. It follows at once that, if $E$ has an isolated point, $c$ say, then $a<c<b, c$ is an AC-point of $F$, and $F$ has a strict local extremum at $c$, but if $E$ is empty, then $F$ is strictly monotonic and $\mathrm{AC}$ on $I$.

Suppose now $E$ is non-empty and perfect. Then, since by (ii) $F$ is (ACG) on $I$, by Baire's theorem $F$ must be AC on some perfect portion $E \cap[p, q]$ of $E$.

Since, as shown above, $F$ is monotone and $\mathrm{AC}$ on each closed interval contiguous to $E$ in $[p, q]$, it easily follows that $F$ is $\mathrm{VB}$ and continuous on $[p, q]$. Hence by (iii) $F$ is $\mathrm{AC}$ on $[p, q]$. Also, since $E \cap] p, q[\neq \emptyset, F$ is not strictly monotone on $[p, q]$ and hence $F$ must have a local extremum at some point $c \in] p, q[$.

The above proof of (3.2)(ii) contains the germ of

Theorem 3.3 Suppose $T$ satisfies ILP on $I$ and is non-porous on $I$. If $\infty \neq$ $T \bar{F}^{\prime}(x) \leq T \underline{F}^{\prime}(x) \neq-\infty$ for all $x$ in $I$, then $F^{\prime}(x)$ exists finitely for all $x$ in a dense open set in $I$.

Proof. From the proof of (3.2)(ii), $F$ is Lipschitz on each part of some closed $I$-form. So by Baire's theorem, for every $[c, d] \subseteq I$ with $c<d$, there is a perfect portion $[p, q]$ of $[c, d]$ on which $F$ is Lipschitz, say $|F(y)-F(x)| \leq N(y-x)$ for $p \leq x<y \leq q$. Then $G(x)=F(x)+N x$ is non-decreasing on $[p, q]$. So for all $x \in] p, q[$, since $p_{T}(x)=0$, using Note 2.4 for $G$ we clearly have $\bar{G}^{\prime}(x)=T \bar{G}^{\prime}(x)=T \bar{F}^{\prime}(x)+N$ and $\underline{G}^{\prime}(x)=T \underline{G}^{\prime}(x)=T \underline{F}^{\prime}(x)+N$. Since $\infty \neq T \bar{F}^{\prime}(x) \leq T \underline{F}^{\prime}(x) \neq-\infty$, it follows that $G^{\prime}(x)$ exists finitely for all $x$ in $] p, q\left[\right.$. Hence $F^{\prime}(x)=G^{\prime}(x)-N$ exists finitely for all $x$ in $] p, q[$.

We are now ready to analyze the Darboux property of derivatives.

Theorem 3.4 Hypotheses: T satisfies ILP on $I, T \bar{F}^{\prime}(x)<\infty$ and $T \underline{F}^{\prime}(x)>-\infty$ for all $x$ in $I$, the set $D$ of points of $I$ where $F$ is $T$-derivable contains at least the $A C$ points of $F$ in $] a, b\left[, D_{*}=\left\{x \in D ; T F^{\prime}(x)\right.\right.$ exists $\}, D_{a c}=\left\{x \in D_{*}\right.$ and $x$ is an AC-point of $F$ in $] a, b[\}$. Conclusions:

(i) If $T \bar{F}^{\prime}(p)<\alpha<T \underline{F}^{\prime}(q)$ for some $\alpha \in \mathbb{R}$ and $p, q \in I$, with possibly $p=q$, then for every $] u, v[\subset I$ with $p, q \in[u, v], F$ has an AC-point $d \in] u, v\left[\right.$ where $T F^{\prime}(d)$ exists, so $d \in D_{a c}$, and equals $\alpha$. 
(ii) If $D_{a c} \subseteq E \subseteq D$, then every extended real-valued function $g$, satisfying $T \bar{F}^{\prime}(x) \leq$ $g(x) \leq T \underline{F}^{\prime}(x)$ for all $x$ in $E$ is Darboux on $E$; in particular both $T \bar{F}^{\prime}$ and $T \underline{F}^{\prime}$ are Darboux on $E$.

(iii) If $D_{a c} \subseteq E \subseteq D_{*}$, then $T F^{\prime}$ is Darboux on $E$.

Proof. Clearly (i) implies both (ii) and (iii).

To prove (i) let $G(x)=F(x)-\alpha x$ for all $x \in I$. Then $T \bar{G}^{\prime}(p)=T \bar{F}^{\prime}(p)-\alpha<0$ and $T G^{\prime}(q)=T F^{\prime}(q)-\alpha>0$, which together imply that $G$ is not monotone on $[u, v]$. Since $T \bar{G}^{\prime}(x)=T \bar{F}^{\prime}(x)-\alpha<\infty$ and $T \underline{G}^{\prime}(x)=T \underline{F}^{\prime}(x)-\alpha>-\infty$ for all $x \in[u, v]$, it follows from (3.2)(iv) that there is a $d \in] u, v[$, an AC-point of $G$, (hence also of $F)$, such that $G(d)$ is a local extremum of $G$; so $T \bar{G}^{\prime}(d) \geq 0$ and $T \underline{G}^{\prime}(d) \leq 0$. Since by hypothesis $F$ is $T$-derivable at $d$, we get

$$
0 \leq T \bar{G}^{\prime}(d)=T \bar{F}^{\prime}(d)-\alpha \leq T \underline{F}^{\prime}(d)-\alpha=T \underline{G}^{\prime}(d) \leq 0 .
$$

Hence it follows that $T F^{\prime}(d)$ exists with value $\alpha$.

A similar result to this is the desired mean value property.

Theorem 3.5 Under the hypotheses of (3.4), $F$ has an AC-point $c \in] a, b[$ where $T F^{\prime}(c)$ exists and equals $r=(F(b)-F(a)) /(b-a)$.

Proof. This follows from the preceding proof, since now $G(x)=F(x)-r x$ is not strictly monotone on $I$ because $G(a)=G(b)$.

Corollary 3.6 If in (3.4) it is assumed further that $T F^{\prime}(x) \geq 0$ for all $x$ in $D_{a c}$, then $F$ is non-decreasing on $I$.

Next we prove the semi-Baire-1 property, in a form which also gives a strengthened version of the O'Malley property [8].

Theorem 3.7 Suppose $T$ satisfies ILP on $I$ and is (NPG) in I, and $T \bar{F}^{\prime}(x) \leq$ $f(x) \leq T \underline{F}^{\prime}(x)$ for all $x$ in $I$. Then $f$ is semi-Baire-1 on $I$.

In fact, if $E$ is the closure of $f^{-1}(f(t))$ for any $t \in I$, then $F$ has an AC-point $c$ in $I, a \leq t \leq c<b$ or $a<c \leq t \leq b$, such that $f(c)=f(t)$ and $F^{\prime}(c)$ exists, (and so does $\left.T F^{\prime}(c)\right)$, with the value $f(c)=f(t)$, and all of the functions $f, T \bar{F}^{\prime}, T \underline{F}^{\prime}, \bar{F}^{\prime}, \underline{F}^{\prime}$ are continuous at $c$ relative to $E$.

Proof. We can assume without loss in generality that $f(t)=0$, for otherwise we could consider the functions $F(x)-f(t) x$ and $f(x)-f(t)$. Now $F$ is $T$-derivable on $I$, and $T \bar{F}^{\prime}(x)<\infty$ and $T \underline{F}^{\prime}(x)>-\infty$ for all $x$ in $I$ since $f(x)$ is a finite function. So by (3.4)(ii) $f$ is Darboux on $I$. 
First suppose $E$ has no perfect portion. Then $E$ must have an isolated point $c$, such that either $a \leq t \leq c<b$ or $a<c \leq t \leq b$, and then $f(c)=f(t)=0$. Continuity at $c$ relative to $E$ is then trivial. Also, let $a \leq c<d \leq b$ where $E \cap] c, d]=\emptyset$. Since $f^{-1}(0) \subseteq E$, the Darboux property of $f$ implies that for all $\left.x \in\right] c, d[$, either $T \bar{F}^{\prime}(x) \leq f(x)<0$ or $0<f(x) \leq T \underline{F}^{\prime}(x)$. Hence by (2.3) and (3.1) $F$ is monotone on $[c, d]$, and hence by $(3.2)(\mathrm{i})$, (iii) $F$ is $\mathrm{AC}$ on $[c, d]$. Besides, $p_{T}(c)<1$ since $T$ is (NPG) on $I$, and $T \bar{F}^{\prime}(c) \leq f(c)=0 \leq T \underline{F^{\prime}}(c)$. Hence from (2.4) we get $F_{+}^{\prime}(c)=0$. By symmetry, if $a<c \leq b$, then $F$ is $\mathrm{AC}$ on some $[d, c], d<c$, and $F_{-}^{\prime}(c)=0$. Thus $c$ is an AC-point of $F$ on $I$ and $F^{\prime}(c)=0=f(t)$.

Next suppose that $E$ has a perfect portion. Then since $T$ is (NPG) on $I$ and, by (3.2)(ii), $F$ is (ACG) on $I$, so by Baire's theorem there must exist a perfect portion $E_{0}$ of $E$ and $0<q<1$, such that $p_{T}(x)<q$ for all $x \in E_{0}$, and $F$ is $\mathrm{AC}$ on $E_{0}$.

Now, given an $\epsilon>0$ let $\eta=\epsilon(1-q)^{2} / 3$. Then there are $T$-full covers $\tau, \lambda$ of $I$ such that for all $x \in I$ we have

$$
\begin{array}{ll}
F(v)-F(u) \leq(f(x)+\eta)(v-u) & \text { for all }[u, v] \in I_{\tau(x)}, \\
F(v)-F(u) \geq(f(x)-\eta)(v-u) & \text { for all }[u, v] \in I_{\lambda(x)} .
\end{array}
$$

Let $\tau$ and $\lambda$ satisfy ILP on $I$ via $\left\{\left(A_{n}, \delta_{n}\right)\right\}$ and $\left\{\left(B_{n}, \rho_{n}\right)\right\}$ respectively. Then $I$ is the union of the sets

$$
E_{n, i}=A_{n} \cap B_{n} \cap f^{-1}([i \eta,(i+1) \eta]), n=1,2, \ldots, i=0, \pm 1, \pm 2, \ldots .
$$

So by Baire's theorem some $E_{n, i}$ must be dense in some perfect portion of $E_{0}$ which we can take as $E_{0} \cap[r, s]=E \cap[r, s]$ with $0<s-r<\min \left\{\delta_{n}, \rho_{n}\right\}$. Since $f^{-1}(0) \subseteq E$, it follows as before that $F$ is monotone and $\mathrm{AC}$ on each closed interval contiguous to $E$ in $[r, s]$, and hence by (3.2)(iii) $F$ is AC on $[r, s]$.

Again, let $x, y \in E_{n, i} \cap[r, s], x<y$. By ILC of $\tau$ and (2.1), given $\delta>0$ there is a $\tau$-partition $\{([x, u], x),([v, y], y)\} \cup \varpi$ of $[x, y]$, where $\varpi$ is a $\tau$-partition of $[u, v]$ with $\sum(|f|, \varpi)<\delta$ and $v-u<\delta$. Then

$$
\begin{aligned}
F(y)-F(x) & =(F(u)-F(x))+\Delta(F, \varpi)+(F(y)-F(v)) \\
& \leq(f(x)+\eta)(u-x)+\sum(f, \varpi)+\eta(v-u)+(f(y)+\eta)(y-v) \\
& <(i+2) \eta(u-x)+\delta+\eta \delta+(i+2) \eta(y-v) \\
& \leq(i+2) \eta(y-x)+|i+2| \eta \delta+(1+\eta) \delta .
\end{aligned}
$$

Letting $\delta \rightarrow 0+$ we get that $F(y)-F(x) \leq(i+2) \eta(y-x)$. Similarly from ILC of $\lambda$ we get that $F(y)-F(x) \geq(i-1) \eta(y-x)$.

Hence, since the set $E_{n, i}$ is dense in $E \cap[r, s]$ and $F$ is continuous on $[r, s]$, for all distinct $x, y \in E \cap[r, s]$ we have

$$
(i-1) \eta \leq \frac{F(y)-F(x)}{y-x} \leq(i+2) \eta .
$$


Now, since $E$ is the closure of $f^{-1}(0)$ and $\left.E \cap\right] r, s[\neq \emptyset$, there is an $e \in E \cap] r, s[$ such that $f(e)=0$. Then $T \bar{F}^{\prime}(e) \leq 0 \leq T \underline{F}^{\prime}(e)$ and $p_{T}(e)<1$. Hence by (5) and (2.4) we get

$$
\frac{(i-1) \eta}{1-p_{T}(e)} \leq 0 \leq \frac{(i+2) \eta}{1-p_{T}(e)}
$$

So $-2 \leq i \leq 1$. Hence by (1), for all distinct $x, y \in E \cap[r, s]$ we have

$$
-3 \eta \leq \frac{F(y)-F(x)}{y-x} \leq 3 \eta \text {. }
$$

Hence by (2.4), for all $x \in E \cap[r, s]$, if $T \underline{F}^{\prime}(x) \geq 0$, then $T \bar{F}^{\prime}(x) \leq T \underline{F}^{\prime}(x)<$ $3 \eta /(1-q)$, and if $T \bar{F}^{\prime}(x) \leq 0$, then $T \underline{F}^{\prime}(x) \geq T \bar{F}^{\prime}(x)>-3 \eta /(1-q)$, but always

$$
\frac{\min \left\{-3 \eta, T \underline{F}^{\prime}(x)\right\}}{1-p_{T}(x)} \leq \underline{F}^{\prime}(x) \leq \bar{F}^{\prime}(x) \leq \frac{\max \left\{3 \eta, T \bar{F}^{\prime}(x)\right\}}{1-p_{T}(x)} .
$$

Hence for all $x \in E \cap[r, s]$, considering all possible signs of $T \underline{F}^{\prime}(x)$ and $T \bar{F}^{\prime}(x)$ we get $-3 \eta /(1-q)^{2}<\underline{F}^{\prime}(x) \leq \bar{F}^{\prime}(x)<3 \eta /(1-q)^{2}$. Thus $-\epsilon<\underline{F}^{\prime}(x) \leq \bar{F}^{\prime}(x)<\epsilon$ for all $x \in E \cap[r, s]$.

Consequently, taking $\epsilon$ to be $1,1 / 2,1 / 3, \ldots$ in succession, we can find intervals $\left[r_{n}, s_{n}\right]$ with end points in $E$, such that $r_{n}<r_{n+1}<s_{n+1}<s_{n}<r_{n}+n^{-1}, F$ is $\mathrm{AC}$ on $\left[r_{1}, s_{1}\right]$, and $-n^{-1}<\underline{F}^{\prime}(x) \leq T \bar{F}^{\prime}(x) \leq f(x) \leq T \underline{F}^{\prime}(x) \leq \bar{F}^{\prime}(x)<n^{-1}$ for all $x \in E \cap\left[r_{n}, s_{n}\right]$. Then the point $c=\lim r_{n}=\lim s_{n}$ evidently fulfills all the required conditions .

This permits sharper versions of the Darboux and mean value properties.

Corollary 3.8 Hypotheses: $T$ satisfies $I L P$ and is (NPG) on $I, \infty \neq T \bar{F}^{\prime}(x) \leq$ $T \underline{F}^{\prime}(x) \neq-\infty$ for all $x$ in $I, I_{*}=\left\{x \in I, T F^{\prime}(x)\right.$ exists $\}$ and $I_{a c}=\left\{x \in I_{*}, x\right.$ is an $A C$-point of $F$ in $] a, b\left[\right.$ and $F^{\prime}(x)$ exists $\}$. Conclusions:

(i) If $T \bar{F}^{\prime}(p)<\alpha<T \underline{F}^{\prime}(q)$ for some $\alpha \in \mathbb{R}$ and $p, q \in I$, with possibly $p=q$, then for every $] u, v[\subset I$ with $p, q \in[u, v], F$ has an $A C$-point $d \in] u, v\left[\right.$ where $F^{\prime}(d)$ exists, so $d \in I_{a c}$, and equals $\alpha$.

(ii) If $I_{a c} \subseteq E \subseteq I$, then every extended real-valued function $g$ satisfying $T \bar{F}^{\prime}(x) \leq$ $g(x) \leq T \underline{F}^{\prime}(x)$ for all $x \in E$, is Darboux on $E$.

(iii) If $I_{a c} \subseteq E \subseteq I_{*}$, then $T F^{\prime}$ is Darboux on $E$.

(iv) There is a $d \in I_{a c}$ such that $F(b)-F(a)=(b-a) F^{\prime}(d)$.

Proof. Clearly (i) implies (ii) and (iii). Now, assume that $T \bar{F}^{\prime}(x) \leq f(x) \leq T \underline{F}^{\prime}(x)$ for all $x \in I$. Then by (3.7), for every $c \in] a, b\left[\right.$ there is a $d \in I_{a c}$ such that $F^{\prime}(\bar{d})=f(c)$. 
Since $f(c)=T F^{\prime}(c)$ whenever $T F^{\prime}(c)$ exists, (i) and (iv) follow from (3.4)(i) and (3.5), respectively.

We may show by example that the function $f$ in (3.7) may fail to be Baire-1 even under stronger conditions.

Example 3.9 We shall construct a bounded $F$ having a non-Baire-1 finite path derivative $F_{P}^{\prime}$ on $I$, relative to a bilateral non-porous system of paths $P$ satisfying (I.C.), recall (2.5).

Let $E$ be a non-dense perfect set with bounds $a, b$ and let \{]$a_{n}, b_{n}[\}$ be the sequence of the distinct components of $I \backslash E$. By induction we define distinct sequences of positive integers $\left\{1_{k}\right\},\left\{2_{k}\right\},\left\{3_{k}\right\}, \ldots$ such that

$$
\begin{aligned}
b_{n}<\cdots<b_{n_{2}}<b_{n_{1}}, b_{n_{k}} & \rightarrow b_{n} \text { as } k \rightarrow \infty, \\
\frac{a_{n_{k}}-b_{n_{k+1}}}{a_{n_{k}}-b_{n}} & \rightarrow 0 \text { as } k \rightarrow \infty ;
\end{aligned}
$$

and since they are distinct

$$
m \neq p \Longrightarrow m_{j} \neq p_{k}, j, k=1,2, \ldots
$$

First observe the following construction: for any $n$ and any $d_{n}>b_{n}$ there is $\left.c_{1} \in E \cap\right] b_{n}, d_{n}\left[\right.$. Let $c_{j}=b_{n}+\left(c_{1}-b_{n}\right) / j, j=2,3, \ldots$ Then $b_{n}<\cdots<c_{2}<c_{1}$, and $c_{j} \rightarrow b_{n}$ as $j \rightarrow \infty$. We can select $n_{1}$ such that $\left.\left.b_{n_{1}} \in\right] c_{2}, c_{1}\right]$, and then select the integers $n_{k+1}$ successively such that $] a_{n_{k+1}}, b_{n_{k+1}}[$ intersects $] c_{2+j_{k}}, c_{1+j_{k}}[$, where $j_{k}$ is the unique index such that $\left.\left.a_{n_{k}} \in\right] c_{1+j_{k}}, c_{j_{k}}\right]$. Clearly $\left\{n_{k}\right\}$ satisfies (6), and it also satisfies (7) because

$$
0<\frac{a_{n_{k}}-b_{n_{k+1}}}{a_{n_{k}}-b_{n}}<\frac{c_{j_{k}}-c_{2+j_{k}}}{c_{1+j_{k}}-b_{n}}<\frac{2}{j_{k}} \rightarrow 0 \quad \text { as } \quad k \rightarrow \infty .
$$

Now, taking $n=1$, so $d_{1}>b_{1}$, take $d_{1}=b$ say, we construct a sequence as above and call it $\left\{1_{k}\right\}$. Suppose then for some $n \geq 2$ and $p=1,2, \ldots, n-1$ the sequences $\left\{p_{k}\right\}$ have been defined so as to satisfy (6), (7) and (8) among them. Evidently we can find $d_{n}>b_{n}$ such that $] b_{n}, d_{n}\left[\right.$ does not contain any of the points $b_{p_{k}}$ for $p=$ $1,2, \ldots, n-1$ and $k=1,2, \ldots$. Then we define $\left\{n_{k}\right\}$ as above with $\left.\left\{b_{n_{k}}\right\} \subset\right] b_{n}, d_{n}[$. This completes the induction.

Now, for each $n$ we define a strictly increasing two-way sequence $\left\{t_{n, i}\right\}_{i=-\infty}^{\infty}$ in $] a_{n}, b_{n}[$ as follows:

$$
\begin{aligned}
& t_{n, i}=a_{n}+\frac{n\left(b_{n}-a_{n}\right)}{2(n-i)} \text { for } i=0,-1,-2, \ldots, \\
& t_{n, i}=b_{n}-\frac{n\left(b_{n}-a_{n}\right)}{2(n+i)} \text { for } i=1,2, \ldots
\end{aligned}
$$


So $t_{n,-j} \rightarrow a_{n}$, and $t_{n, j} \rightarrow b_{n}$ as $j \rightarrow \infty$. Also we have

$$
\max \left\{\frac{t_{n, i+5}-t_{n, i}}{t_{n, i+5}-a_{n}}, \frac{t_{n, i+5}-t_{n, i}}{b_{n}-t_{n, i}}\right\} \leq \frac{5}{n+|i|} .
$$

Let $\left.\left.I_{n, r}=\cup_{i=-\infty}^{\infty}\right] t_{n, 6 i+r}, t_{n, 6 i+r+1}\right]$ for $r=0,1, \ldots, 5$ only. Then $I_{n, r} \cap I_{n, s}=\emptyset$ for $r \neq s$. We define

$$
P_{x}= \begin{cases}(\mathbb{R} \backslash I) \cup E \cup \bigcup_{n=1}^{\infty} I_{n, 0} & \text { if } x \in E, x \notin\left\{b_{n}\right. \\ \{x\} \cup I_{n, 2} \cup \bigcup_{k=1}^{\infty} I_{n_{k}, 4} & \text { if } x=b_{n}, n=1,2, \ldots, \\ \mathbb{R} & \text { if } x \in \mathbb{R} \backslash E .\end{cases}
$$

Using (6), (7) and (9) we readily verify that $P=\left\{P_{x} ; x \in \mathbb{R}\right\}$ is a bilateral, nonporous system of paths.

Also, if $\delta: \mathbb{R} \rightarrow] 0,1\left[\right.$ is such that $\delta\left(b_{n}\right)<\min \left\{\left|b_{1}-b_{n}\right|,\left|b_{2}-b_{n}\right|, \ldots,\left|b_{n-1}-b_{n}\right|\right\}$ for $n \geq 2$, then $\left|b_{m}-b_{n}\right|>\min \left\{\delta\left(b_{m}\right), \delta\left(b_{n}\right)\right\}$ for all $m \neq n$ and hence, clearly $P$ satisfies (I.C.) with respect to $\delta$.

Now, recalling (3), for each $n$ we define $n^{*}=m$ if $n=m_{k}$ for some $m$ and $k$, and $n^{*}=n$ otherwise. Then we define

$$
F(x)= \begin{cases}0 & \text { if } x \in E \cup \bigcup_{n=1}^{\infty} I_{n, 0}, \\ x-b_{n} & \text { if } x \in I_{n, 2}, n=1,2, \ldots, \\ x-b_{n^{*}} & \text { if } x \in I_{n, 4}, n=1,2, \ldots\end{cases}
$$

Also we define $F(x)$ on each of $I_{n, 1}, I_{n, 3}, I_{n, 5}$ in such a way that $F$ becomes differentiable on each of the intervals $] a_{n}, b_{n}[$, and remains bounded on $I$. Then we see at once that $F_{P}^{\prime}(x)$ exists finitely for all $x$ in $I$. (Note that $\left(n_{k}\right)^{*}=n$.) But $F_{P}^{\prime}\left(b_{n}\right)=1$ for all $n$ and $F_{P}^{\prime}(x)=0$ for all $x \in E, x \notin\left\{b_{n}\right\}$, so $F_{P}^{\prime}$ has no points of continuity in $E$ relative to $E$ since $\left\{a_{n}\right\}$ and $\left\{b_{n}\right\}$ are disjoint dense subsets of $E$. Hence $F_{P}^{\prime}$ is not Baire-1 on I, though by (3.7) it is semi-Baire-1 on I.

Our next example shows that the O'Malley property is non-trivial insofar as it may fail even for strictly increasing absolutely continuous $F$ and for $T$ satisfying (IC), in the absence of the condition (NPG).

Example 3.10 It is not difficult to find a strictly increasing absolutely continuous $F$, such that $0<F^{\prime}(x)<\infty$ for all $x$ in $\left.] a, b\right]$ but with $0=D_{+} F(a)<D^{+} F(a)<\infty$. Then there is a strictly decreasing sequence $\left\{a_{n}\right\}$ in ] $a, b[$ converging to a such that $\left(F\left(a_{n}\right)-F(a)\right) /\left(a_{n}-a\right) \rightarrow 0$. We define a bilateral system of paths $P$ by setting $P_{x}=\mathbb{R}$ for $x \neq a$, and $\left.\left.P_{a}=\right]-\infty, a\right] \cup\left\{a_{n}\right\}$. Obviously $P$ satisfies (I.C.), and $F_{P}^{\prime}(x)$ exists finitely for all $x$ in $I$ with $F_{P}^{\prime}(a)=0$. But the O'Malley property fails at a, since $F^{\prime}(a)$ does not exist and $F^{\prime}(x)>0$ for $a<x \leq b$. 
Next, we give a proof of a stronger version of the Weil property [17], inclusive of the Denjoy property.

Theorem 3.11 Suppose T satisfies ILP on I and is (NPG) on I, and $T \bar{F}^{\prime}(x) \leq f(x)$ $\leq T \underline{F}^{\prime}(x)$ for all $x$ in $I$. Then for every $t \in I$ and $\alpha<f(t)<\beta$, every one-sided neighborhood of $t$ in I contains an interval $J$ on which $F$ is $A C,\left(\right.$ so $f(x)=F^{\prime}(x)$ a.e. on $J)$, such that $\left|J \cap f^{-1}(] \alpha, \beta[)\right|>0$.

Proof. Let $E$ denote the closure of $f^{-1}(f(t))$, and suppose that $a \leq t<v<b$. By (3.7), $F$ has an AC-point $c$ in $[t, v[$ such that $f(c)=f(t)$, (so $\alpha<f(c)<\beta)$ and both $T \bar{F}^{\prime}, T \underline{F}^{\prime}$ are continuous at $c$ relative to $E \cap[t, v[$. Then let $c<d<v$ be such that $F$ is $\mathrm{AC}$ on $J_{0}=[c, d]$, and

$$
\alpha<T \bar{F}^{\prime}(x) \leq f(x) \leq T \underline{F}^{\prime}(x)<\beta \text { for all } x \in E \cap J_{0} .
$$

If $\left|E \cap J_{0}\right|>0$, then by (10) $\left|J \cap f^{-1}(] \alpha, \beta[)\right|>0$ with $J=J_{0}$.

Suppose that $\left|E \cap J_{0}\right|=0$. Let $J=[r, s]$ be the closure of a component of $J_{0} \backslash E$. Since $c \in E$, clearly $r \in E \cap J_{0}$. So by $(10) D^{+} F(r) \geq T \underline{F}^{\prime}(r)>\alpha$, and $D_{+} F(r) \leq T \bar{F}^{\prime}(r)<\beta$, which by (3.1)(i), (ii) imply, respectively

$$
\left|\left\{x \in J ; T \bar{F}^{\prime}(x)>\alpha\right\}\right|>0 \text { and }\left|\left\{x \in J ; T \underline{F}^{\prime}(x)<\beta\right\}\right|>0 .
$$

Since $T \bar{F}^{\prime}(x) \leq f(x) \leq T \underline{F}^{\prime}(x)$ for all $x$ in $J=[r, s]$ we get

$$
|\{x \in J ; f(x)>\alpha\}|>0 \text { and }|\{x \in J ; f(x)<\beta\}|>0 .
$$

But, since $] r, s\left[\cap f^{-1}(f(t))=\emptyset\right.$, the Darboux property of $f$, (3.4)(ii), implies that either $f(x)<f(t)<\beta$ or $f(x)>f(t)>\alpha$ for $x \in] r, s[$. Hence, by (11), in either case $\left|J \cap f^{-1}(] \alpha, \beta[)\right|>0$.

Similarly, if $a<u<t \leq b$, we can find a $J$ in some $[d, c] \subset] u, t]$, and this completes the proof of the theorem.

Finally we will say that a function $f$ has the property $Z^{*}$ on $I$, if for every $c \in I$ and $\epsilon>0, \eta>0$ there is a neighborhood $I_{c}$ of $c$ in $I$ such that the following conditions $Z^{+}, Z^{-}$hold.

$Z^{+}$: If $f(x) \geq f(c)-\epsilon$ a.e. on a closed interval $J \subset I_{c}$ then $|A|-|B| \leq \eta \rho(c, J)$, where $A=\{x \in J ; f(x) \geq f(c)+\epsilon\}$ and $B=\{x \in J ; f(c)-\epsilon \leq f(x)<f(c)\}$, and $\rho(c, J)=\max \{|x-c| ; x \in J\}$.

$Z^{-}$: If $f(x) \leq f(c)+\epsilon$ a.e. on a closed interval $J \subset I_{c}$ then $|A|-|B| \leq \eta \rho(c, J)$, where $A=\{x \in J ; f(x) \leq f(c)-\epsilon\}$ and $B=\{x \in J ; f(c)<f(x) \leq f(c)+\epsilon\}$.

We remark that $f$ satisfies $Z^{-}$if and only if $-f$ satisfies $Z^{+}$. In another paper it will be shown that property $Z^{*}$ is strictly stronger than the Zahorski-Weil property $Z$; $([18$, p. 528$]$, has a misprint of $\leq \epsilon$ for $\geq \epsilon)$, and that every approximate Peano derivative has the property $Z^{*}$. 
Theorem 3.12 If $T$ satisfies ILP on $I$ and is non-porous on $I$ and $T \bar{F}^{\prime}(x) \leq f(x) \leq$ $T \underline{F}^{\prime}(x)$ for all $x$ in $I$, then $f$ has the property $Z^{*}$ on $I$.

Proof. Considering $-f$ and $-F$ clearly we need only prove $Z^{+}$for $f$.

Fix $c \in I, \epsilon>0, \eta>0$, put $G(x)=F(x)-F(c)-(x-c) f(c)$ and $g(x)=f(x)-f(c)$ for $x$ in $I$. Then $G(c)=g(c)=0$, and

$$
T \bar{G}^{\prime}(x)=T \bar{F}^{\prime}(x)-f(c) \leq g(x) \leq T \underline{F}^{\prime}(x)-f(c)=T \underline{G^{\prime}}(x), \quad x \in I .
$$

Since $T \bar{G}^{\prime}(c) \leq 0 \leq T \underline{G}^{\prime}(c)$, there are $\tau(c), \lambda(c) \in T(c)$ such that

$$
\frac{G(q)}{q-c} \begin{cases}<\frac{\epsilon \eta}{4} & \text { if } c \neq q \in I \cap \tau(c), \\ >-\frac{\epsilon \eta}{4} & \text { if } c \neq q \in I \cap \lambda(c) .\end{cases}
$$

Since $p_{T}(c)=0$, there is a neighborhood $I_{c}$ of $c$ in $I$ such that

$$
\left.s-r \leq \frac{\eta}{4} \rho(c,[r, s]) \text { if }\right] r, s\left[\subset I_{c} \backslash \tau(c) \quad \text { or } \quad\right] r, s\left[\subset I_{c} \backslash \lambda(c) .\right.
$$

We show that for every $J=[x, y] \subset I_{c}$ for which $G \mid J$ is continuous, there is a $[u, v] \subseteq J$ such that

$$
u-x+y-v \leq \frac{\eta}{2} \rho(c, J) \text { and } G(v)-G(u) \leq \frac{\epsilon \eta}{2} \rho(c, J) .
$$

If $c<x \leq y$, take $v=\inf \{t \in[x, y] ;] t, y[\cap \tau(c)=\emptyset\}$ and $u=\sup \{t \in[x, v] ;] x, t[\cap \lambda(c)=$ $\emptyset\}$. Note that, if $u \neq v$, then $v$ and $u$ belong to the closures of $\lambda(c) \cap[u, v]$ and $\tau(c) \cap[u, v]$, respectively.

If $x \leq y<c$ take $v=\inf \{t \in[x, y] ;] t, y[\cap \lambda(c)=\emptyset\}$ and $u=\sup \{t \in[x, v] ;] x, t[\cap \tau(c)=$ $\emptyset\}$. Note that, if here $u \neq v$, then $v$ and $u$ belong to the closures of $\lambda(c) \cap[u, v]$ and $\tau \cap[u, v]$ respectively.

If $x \leq c \leq y$ take $v=\sup ([c, y] \cap \tau(c))$ and $u=\inf ([x, c] \cap \tau(c))$. Note that, now both of $u, v$ belong to the closure of $\tau(c) \cap[u, v]$.

In all cases, the first part of (14) follows at once from (13), and the second part follows by noting that, if $u \neq v$, then continuity of $G \mid[u, v]$ implies by (12) that $G(v) \leq(\epsilon \eta / 4)|v-c|$ and $-G(u) \leq(\epsilon \eta / 4)|u-c|$.

Now, let $f(x) \geq f(c)-\epsilon$ a.e. on some $J=[x, y] \subset I_{c}$. With $A, B$ as in $Z^{+}$, we have $A=\{x \in J ; g(x) \geq \epsilon\}$, and $B=\{x \in J ;-\epsilon \leq g(x)<0\}$.

Since $T \underline{G}^{\prime}(x) \geq g(x) \geq-\epsilon$ a.e. on $J$, by (3.1) $G(x)+\epsilon x$ is non-decreasing on $J$. Hence, clearly, by (3.2)(i), (iii) $G$ is AC on $J$. So there is a $[u, v] \subseteq J$ satisfying (14), and since $g(x)=G^{\prime}(x)$ a.e. on $J$ and $g(x) \geq 0$ a.e. on $J \backslash A \cup B$, we have, further

$$
G(v)-G(u)=\int_{u}^{v} g \geq \epsilon|A \cap[u, v]|-\epsilon|B \cap[u, v]| .
$$


Since $|A|-|B| \leq u-x+y-v+|A \cap[u, v]|-|B \cap[u, v]|$, from (14) and (15) it follows that $|A|-|B| \leq \eta \rho(c, J)$.

Thus $f$ has the property $Z^{+}$on $I$, and we are finished.

\section{References}

[1] A. M. Bruckner and J. G. Ceder, Darboux continuity, Jber. Deutsch. Math. Verein, 67 (1965), 93-117.

[2] A. M. Bruckner, R. J. O'Malley and B. S. Thomson, Path derivatives: A unified view of certain generalized derivatives, Trans. Amer. Math. Soc., 283 (1984), 97-125.

[3] P. S. Bullen, Some applications of partitioning covers, Real Analysis Exch., 9 (1983-1984), 539-557.

[4] P. S. Bullen and D. N. Sarkhel, On Darboux and mean value properties, Canad. Math. Bull., 30 (1987), 223-230.

[5] C. Goffman and C. J. Neugebauer, On approximate derivatives, Proc. Amer. Math. Soc., 11 (1960), 962-966.

[6] E. W. Hobson, The Theory of Functions of a Real Variable and the Theory of Fourier Series, Vol. 1, Dover, New York, 1957.

[7] A. Khintchine, Recherches sur la structure des fonctions mesurables, Fund. Math., 9 (1927), 217-279.

[8] R. J. O'Malley, The set where an approximate derivative is a derivative, Proc. Amer. Math. Soc., 54 (1976), 122-124.

[9] S. Marcus, On a theorem of Denjoy and on approximate derivative, Monatsh. Math., 66 (1962), 435-440.

[10] S. Saks, Theory of the Integral, Dover, New York, 1964.

[11] D. N. Sarkhel and A. B. Kar, (PVB) function and integration, J. Austral. Math. Soc., Ser. A, 36 (1984), 335-353.

[12] D. N. Sarkhel and P. K. Seth, On some generalized approximative relative derivatives, Rendiconti Circolo Mat. Palermo, 35(2) (1986), 5-21.

[13] H. K. Sen, Darboux's property and its applications, Proc. Benares Math. Soc., (N.S.) 2 (1940), 17-23. 
[14] B. S. Thomson, On full covering properties, Real Analysis Exch., 6 (1980-1981), 77-93.

[15] B. S. Thomson, Derivation bases on the real line, II, Real Analysis Exch., 8 (1982-1983), 278-442.

[16] G. P. Tolstoff, Sur la dérivée approximative exacte, Rec. Math. (Mat. Sb.), (N.S.) 4 (1938), 499-504.

[17] C. E. Weil, On approximate and Peano derivatives, Proc. Amer. Math. Soc., 20 (1969), 487-490.

[18] C. E. Weil, A property for certain derivatives, Indiana Univ. Math. J., 23 (197374), 527-536.

[19] Z. Zahorski, Sur la prémière dérivée, Trans. Amer. Math. Soc., 69 (1950), 1-54. 